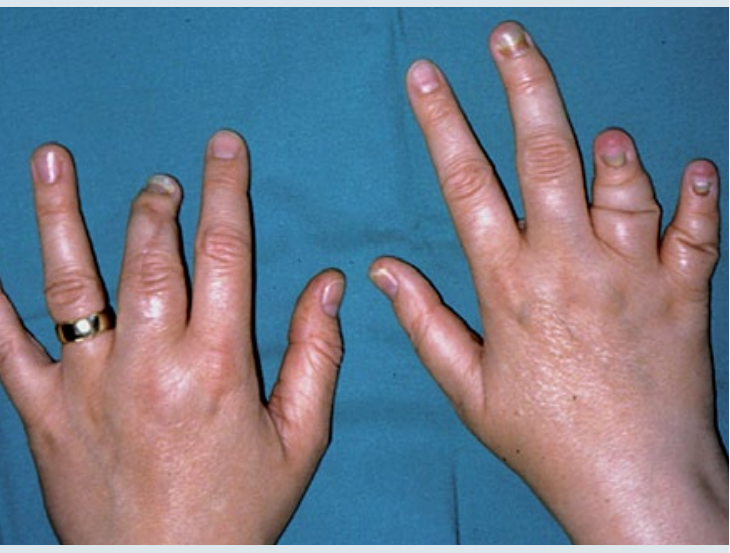

\section{CME: Psoriasisarthritis}

Bei bis zu $25 \%$ der Psoriasis-Patienten zeigt sich früher oder später auch eine entzündliche Gelenkerkrankung. Wie eine Psoriasisarthritis diagnostiziert und behandelt wird, ist Thema unserer CME.

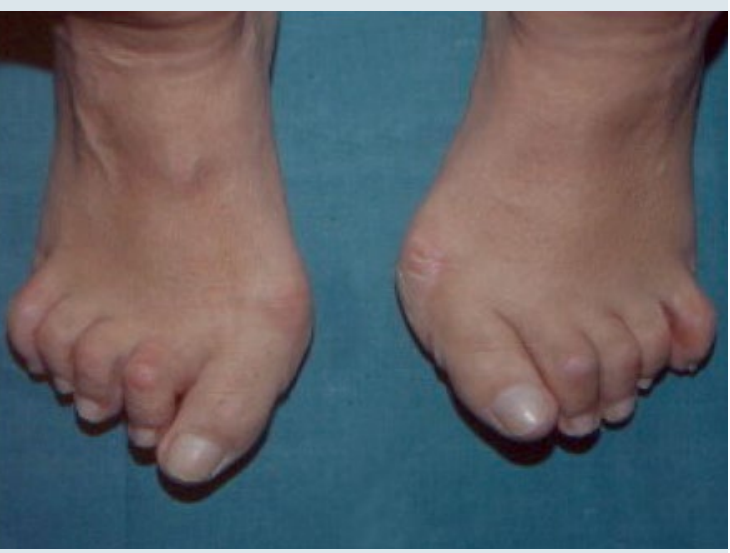

31 OP-Optionen für Rheumafüße

Das Schlüsselgelenk für die Entwicklung einer rheumatischen Vorfußdeformität ist das Metatarsophalangealgelenk. Ist eine operative Revision erforderlich, sollte man die OP-Technik den individuellen

Gegebenheiten anpassen.
Panorama

8 Nachrichten aus Medizin und Politik

\section{Literatur Kompakt}

\section{Aktuelle Studien kurz referiert}

\section{Im Blickpunkt}

16 Von der Kliniksuche bis zum Antrag auf Schwerbehinderung Mit Rheuma-Lotsen auf sicherem Kurs

\section{Fortbildung}

21 CME: Eine Hautkrankheit und ihre Folgen

Psoriasisarthritis: Manifestationsformen, Diagnose und Therapie D. Schöffel

27 CME-Fragebogen

31 Rezidiv der Großzehenfehlstellung gefährdet den Erfolg Rheumatischer Vorfuß: OP-Technik individuell anpassen T. Patsalis, A Weber

36 Die Prävalenz der Erkrankung ist schwierig zu bestimmen Bisphosphonat-induzierte Nekrose des Kieferknochens: ein kritisches Update C. Pautke, S. Otto

41 Bei ungenügender Qualität drohen Fehldiagnosen Hüftschmerzen: die Fallstricke der Röntgendiagnostik N. Harrasser, A. Rauch

46 Perioperatives Management in der Orthopädie und Unfallchirurgie

Funktionelle interdisziplinäre Therapie macht Patienten schneller fit J. Jeroschi

48 Operationsmikroskop als Meilenstein der Entwicklung Fortschritte der peripheren Nervenchirurgie H. Millesi

\section{Wie Sie uns erreichen}

Redaktion

Dr. med. Brigitta Schneider E-Mail: brigitta.schneider@ springer.com

Telefon 089 203043-1406

Fax 089 203043-1400

Verlag Urban \& Vogel GmbH Aschauer Str. 30

81549 München
Chefredaktion

Dr. med. Michael Pieper

Endocenter Damme

Lindenstraße 10-12

49401 Damme

Prof. Dr. med. Klaus A. Milachowski

Theatinerstr. 35

80333 München

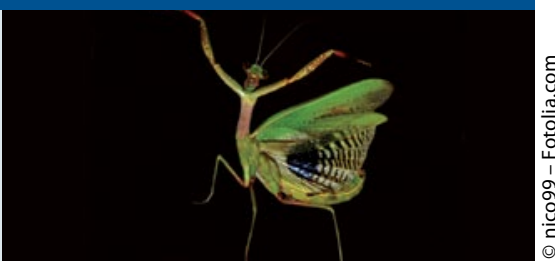

\section{Tango korrupti}

Alle Ärzte sind korrupt und geldgierig. So kolportieren es Massenmedien gern und immer öfter. Öffentliche Verunglimpfung war schon immer ein probates Mittel, um (vermeintlich) Aufmüpfige in die Schranken zu weisen. Das bekommen derzeit auch die Mediziner deutlich zu spüren. 


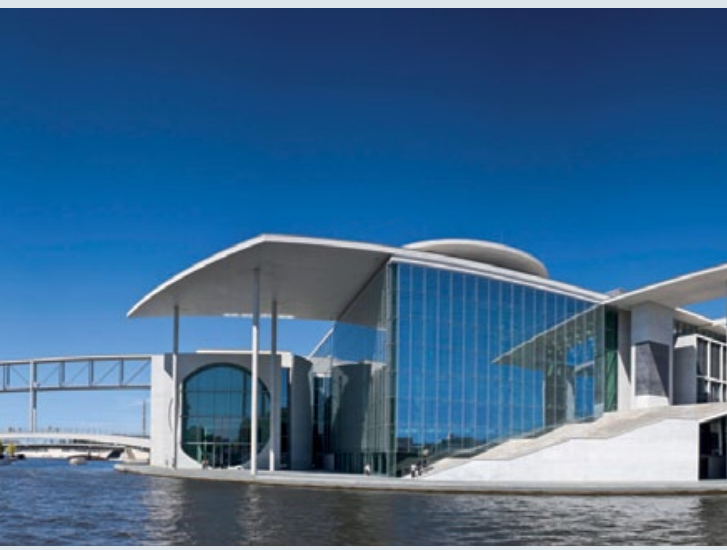

\section{EULAR-Kongress 2012 in Berlin}

Praxisrelevante rheumatologische Fortbildung auf hohem Niveau bot auch in diesem Jahr wieder der Kongress der European League Against Rheumatism. Wir haben einige Highlights für Sie zusammengefasst.

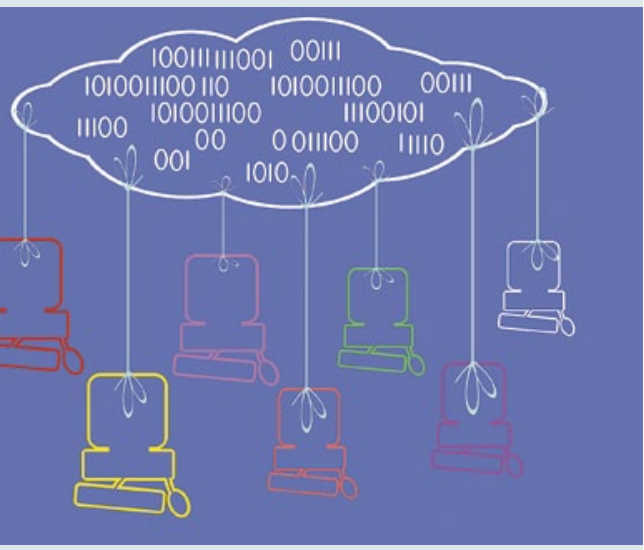

60 Cloud-Computing:

für Arztpraxen nicht ohne Risiken

Die IT-Welt schwärmt vom Cloud-

Computing. Bevor jedoch Ärzte sensible Praxisdaten in externen "Wolken" parken, sollten sie sich sorgfältig über mögliche Risiken in puncto Datenschutz informieren.
IGOST-Mitteilungen

\section{Schmerzspitzen}

Einsichten - Aussichten

53 IGOST-Termine

Medizin aktuell

\section{EULAR 2012}

Kongress-Highlights

56 Deutliche Unterschiede in biomechanischen Tests Thrombosestrumpf ist nicht gleich Thrombosestrumpf

57 Minimalinvasive Therapie von Wirbelkörperfrakturen Pluspunkte für die Radiofrequenzkyphoplastie

\section{Praxis konkret}

\section{Cloud-Computing \\ Die Arztpraxis in der Wolke}

\section{Sicherheit für Patienten}

Wettbewerbsorientiert mit dem richtigen Praxisnotrufsystem

64 Neu-Landarzt berichtet von seinen Erfahrungen Beratung vor Regress - Verlass ist darauf noch nicht

\section{Expertenrat zur Altersvorsorge}

Nutzen Sie Steuervorteile für die Rente!

70 Umfrageergebnisse des KBV-Ärztemonitors

Viel Arbeit, wenig Zeit - trotzdem glücklich

\section{Pharmaforum}

\section{$74 \quad$ Meldungen aus der Industrie}

\section{Prisma}

78 Museum Friedrichsheim zeigt die Entwicklung der Orthopädie Vom „Krüppelheim“ zum Klinikum

80 Der besondere Fall

\section{Zertifizierte Fortbildung}

Wir bieten in jeder Ausgabe eine zertifizierte Fortbildung (CME) an. Den Fragebogen zum aktuellen Beitrag „Psoriasisarthritis: Manifestationsformen, Diagnose und Therapie" finden Sie auf Seite $27 \mathrm{f}$. und unter www.springermedizin.de/eMed.

\section{Beilagenhinweis}

In dieser Ausgabe der Orthopädie \& Rheuma finden Sie folgende Beilagen: IGOST: Update 2012; Wissen \& Gesundheit; Ärztezeitung. Wir bitten um freundliche Beachtung.

\section{Newsletter UPDATE ORTHOPÄDIE}

Alle 14 Tage das Neueste aus Forschung und Berufspolitik bietet Ihnen der Online-Newsletter UPDATE ORTHOPÄDIE. Für diesen kostenlosen Dienst können Sie sich anmelden unter:

www.springermedizin.de/orthopaedieund-rheuma 